\title{
Comunidade de Carabidae (Coleoptera) em manchas florestais no Pantanal, Mato Grosso do Sul, Brasil
}

\author{
Letícia Vieira ${ }^{1}$, Frederico S. Lopes ${ }^{2}$, Wedson D. Fernandes ${ }^{3} \&$ Josué Raizer $^{3}$
}

1. Departamento de Entomologia, Universidade Federal de Lavras, Caixa Postal 3037, 37200-000 Lavras, MG. (leticiacarabidae@yahoo.com.br)

2. Departamento de Biologia, Universidade Federal de Mato Grosso do Sul, Caixa Postal 549, 79070-900 Campo Grande, MS (fslopes@nin.ufms.br)

3. Faculdade de Ciências Biológicas e Ambientais, Universidade Federal da Grande Dourados, Caixa Postal 533, 79825-070 Dourados, MS. (wedson@ufgd.edu.br; jraizer@nin.ufms.br)

\begin{abstract}
Carabid community (Coleoptera) in forest patches in Pantanal, State of Mato Grosso do Sul, Brazil. This work addresses the effects of the size, flooding susceptibility and vegetation complexity on ground beetles community structure (Coleoptera, Carabidae) in forest patches (capões) in the sub-regions Miranda and Abobral of the Pantanal, State of Mato Grosso do Sul. Thirty pitfall traps were run for six days per month, in six forest patches between October 1998 and October 1999. A total of 2,071 individuals of 64 species were captured. Negrea scutellaris (Dejean, 1831) and an unidentified Lebiini were the most abundant species (472 and 464 individuals respectively). Gregarious distribution and great number of species of low abundance could reflect the pattern of resource availability in the tropical regions. Species richness in the forest patches was explained only by vegetation complexity, whereas species composition demonstrated no significant relationship with any of the considered variables. This lack of relationship could have been driven by the limited variation in size and vegetation complexity of capões; alternatively, it may reflect a stronger effect of species composition change at regional, rather than local scales.
\end{abstract}

KEYWORDS. Community structure, Carabidae, Pantanal, vegetation complexity, wetland.

RESUMO. Neste trabalho foi estudado o efeito do tamanho, susceptibilidade à inundação e complexidade vegetacional na estrutura de comunidade de Carabidae (Coleoptera) em ilhas de vegetação arbórea (capões de mata) no Pantanal sul-mato-grossense, sub-regiões Miranda e Abobral. Os dados foram obtidos no período de outubro de 1998 a outubro de 1999 em seis capões de mata, através de 30 armadilhas de queda ("pitfall traps") instaladas por seis dias, mensalmente, no interior dos capões. Foram capturados 2.071 indivíduos, distribuídos em 64 espécies. Negrea scutellaris (Dejean, 1831) e uma espécie não-identificada de Lebiini foram as espécies mais abundantes (com 472 e 464 indivíduos, respectivamente). A distribuição gregária e o elevado número de espécies pouco abundantes encontrados pode refletir o padrão de disponibilidade de recursos das regiões tropicais. Entre as variáveis estudadas, apenas a complexidade vegetacional explicou a variação da riqueza de espécies nos capões de mata. A composição das espécies não foi explicada por nenhuma das variáveis avaliadas. Possivelmente a variação em tamanho e em complexidade vegetacional dos capões não seja tão evidente para demonstrar estas relações e a composição das espécies esteja variando mais em escala regional do que em escala local.

PALAVRAS-CHAVE. Estrutura de comunidade, Carabidae, Pantanal, complexidade vegetacional, planície de inundação.

Carabidae (Coleoptera) é a terceira família mais numerosa de besouros, representada por 40.000 espécies distribuídas em 86 tribos (LöveI \& SuderLand, 1996). A Região Neotropical possui a maior diversidade de espécies (LöVEI \& SudERLAND, 1996) e o Brasil, a maior riqueza (1.506) e ocorrência de gêneros descritos (194) (RoIG-JuÑENT \& Domínguez, 2001).

Os carabídeos são besouros de hábitos diurnos e noturnos (LöveI \& SUDERLAND, 1996). Quanto ao hábito alimentar, podem ser onívoros ou predadores de sementes (Воотн et al., 1990) e de frutos caídos (PAARMANn et al., 2001). Entretanto, a maioria das espécies - tanto as larvas quanto os adultos - é predadora de vários invertebrados (VARCHOLA \& DunN, 1999; LÖVEI \& SuDERLAND, 1996), representando um importante grupo utilizado no manejo de pragas e no controle biológico (PAVUK et al., 1997).

A ocorrência destes besouros pode estar associada com alguns parâmetros ambientais, tais como tipo ou umidade do solo e cobertura vegetal (INGS \& HartLeY, 1999; MolNár et al., 2001; EYrE et al., 2003). São sensíveis a alterações ambientais e climáticas e podem utilizar diferentes ambientes como refúgio em condições desfavoráveis (ThIELE, 1977; VARCHOLA \& DunN, 2001).
As comunidades de carabídeos são moderadamente ricas em espécies, tanto em escala local quanto regional. Geralmente, têm entre 10 e 40 espécies ativas em um mesmo hábitat na mesma estação (LöveI \& SudERLAND, 1996). Muitas espécies são sazonalmente dependentes da paisagem, migrando entre ambientes para a reprodução na primavera e sobrevivência no inverno (VARCHOLA \& DuNN, 1999; Lys et al., 1994). Embora seja um dos grupos taxonômicos mais conhecidos em várias regiões do mundo, a família é pouco estudada na Região Neotropical (Nimielä, 1990a; Diefenbach \& Becker, 1992) e, no Pantanal, os escassos registros correspondem principalmente à descrição de novas espécies (e.g. VIEIRA \& BELLO, 2004).

O Pantanal é uma planície sedimentar formada no período quaternário (SiLVA et al., 2000), que ocupa uma área de aproximadamente $140.000 \mathrm{~km}^{2}$, no centro da América do Sul (Junk, 1993). Esta planície mostra-se extremamente rica na composição físionômica, com formações características, tais como: "vazantes", "corixos", "baías", "cordilheiras" e "capões de mata" termos regionalmente usados para definir corpos d'água e formações vegetais (PonCE \& CunHA, 1993). Os capões de mata - ilhas de floresta semicaducifólia - estão 
situados em terrenos 1 a $3 \mathrm{~m}$ mais elevados que o campo circundante e, geralmente, possuem forma circular ou elíptica (Prance \& Schaller, 1982; Oliveira-Filho, 1992). As áreas mais baixas dos capões de mata estão sujeitas ao alagamento periódico, enquanto as porções mais elevadas não são alagadas, exceto em cheias plurianuais excepcionalmente altas. Em período de inundações normais, os capões de mata apresentam-se como "ilhas" e servem de refúgio para os animais intolerantes à inundação. Assim, espera-se que as comunidades biológicas em capões de mata estejam organizadas segundo a teoria do equilíbrio da biogeografia de ilhas (MACARThur \& Wilson, 1967), como já evidenciado em fragmentos de mata em diferentes regiões (FAHRIG, 2003), mais fortemente em áreas sujeitas à inundação periódica.

A heterogeneidade do hábitat está positivamente relacionada à diversidade de espécies. Mas, dependendo do grupo taxonômico, da escala espacial e do parâmetro estrutural, a diversidade pode até diminuir com o incremento em heterogeneidade (DAVIDOWITZ \& RosENZWEIG, 1998; Sullivan \& Sullivan, 2001; Tews et al., 2004). A comunidade de plantas determina a estrutura física do ambiente para as espécies animais em muitos hábitats (MACARTHUR \& MacArthur, 1961; Lawton, 1983; McCoy \& Bell, 1991; Tews et al., 2004). Em capões com maior complexidade estrutural da vegetação podem ocorrer espécies animais mais especializadas e, portanto, a diversidade de besouros carabídeos deve responder positivamente à complexidade da vegetação, pois a diversidade de nichos na serrapilheira depende da fonte principalmente de folhas e ramos do estrato arbustivo e arbóreo.

Neste trabalho foi avaliado como a estrutura da comunidade de Carabidae (Coleoptera) em capões de mata está relacionada ao tamanho, à inundação e à complexidade da vegetação.

\section{MATERIAIS E MÉTODOS}

Os dados foram obtidos em seis capões de mata no Pantanal sul-mato-grossense, nas sub-regiões Miranda e Abobral, município de Corumbá, Mato Grosso do Sul, proximidades da Base de Estudos do Pantanal (BEP) da Universidade Federal de Mato Grosso do Sul (19³4'36'S; $\left.57^{\circ} 01^{\prime} 06^{\prime \prime} \mathrm{W}\right)$. Um grupo de três destes capões era inundável e outro não. O grupo de capões inundáveis (19³0'10.76"S, 5701'50.41"W; 19³0'3.05"S, 57¹'20.67'W; 19²9'54.70”S, 57¹'41.87’W) está localizado na fazenda São Bento, situada próximo à rodovia MS 184, e o de capões não-inundáveis (19³6'42.66"S, 5657'4.96”W; 19³6'38.82”s,

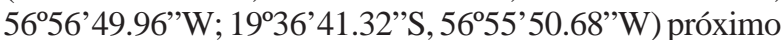
ao rio Vermelho, na fazenda São Judas.

Foram considerados suscetíveis ao alagamento ou inundáveis aqueles capões que possuíam marcas d'água nas árvores, caracterizando um ou mais eventos de invasão da água no seu interior. A área alagada e o tempo de permanência da água nestes capões são aspectos imprevisíveis e variáveis a cada ano; normalmente, os capões próximos ao rio Vermelho não são invadidos pelas águas durante as cheias, por estarem localizados em terrenos mais elevados (91 a $94 \mathrm{~m}$ de altitude) do que os demais capões amostrados ( 89 a 90 m de altitude). Além disso, a altura dos capões em relação ao campo adjacente próximo ao rio Vermelho é de cerca de $3 \mathrm{~m}$, enquanto nos capões próximo à rodovia MS-184 é de aproximadamente $1 \mathrm{~m}$.

Os carabídeos foram coletados mensalmente no período de outubro de 1998 a outubro de 1999. Para a captura dos besouros foram instaladas 30 armadilhas de queda ("pitfall traps") no centro de cada capão de mata, permanecendo no local por um período de seis dias. Elas foram dispostas em três fileiras com dez armadilhas cada, com espaçamento de $1 \mathrm{~m}$ uma da outra e entre as fileiras, formando um retângulo de $18 \mathrm{~m}^{2}$.

As armadilhas foram confeccionadas com potes plásticos de $15 \mathrm{~cm}$ de diâmetro e $10 \mathrm{~cm}$ de altura, contendo $250 \mathrm{ml}$ de líquido conservante - 3/4 do volume de álcool etílico a $70 \%$ e $1 / 4$ de formaldeído a $10 \%$-, acrescido de algumas gotas de detergente doméstico. Todas as armadilhas foram cobertas com pratos plásticos de $20 \mathrm{~cm}$ de diâmetro, a $10 \mathrm{~cm}$ acima da abertura do pote, sustentados por palitos de madeira, para minimizar a entrada da água das chuvas e de restos vegetais.

O material coletado nas armadilhas foi transportado ao laboratório e conservado em álcool $70 \%$ para posterior triagem dos besouros e acondicionamento em mantas de algodão que foram secadas em estufa a $40^{\circ} \mathrm{C}$ por 48 horas Os carabídeos foram montados em alfinetes entomológicos e identificados por meio de chaves dicotômicas (ReIchardt, 1974, 1977; StraneO, 1979; NOONAN, 1985).

As espécies ou morfotipos foram comparados, para confirmação, com exemplares da coleção do Museu de Zoologia da Universidade de São Paulo (MZSP). Exemplares das espécies identificadas neste trabalho estão depositados na coleção de referência do MZSP.

O perímetro dos capões foi obtido em campo com auxílio de trena flexível, como estimativa do tamanho dos capões. A complexidade estrutural da vegetação dos capões foi medida através dos seguintes parâmetros: (1) densidade das partes vegetativas de árvores e (2) de arbustos, obtida através da contagem de estruturas da vegetação que tocavam um transecto de $50 \mathrm{~m}$, partindo do centro de cada capão, de onde foram sorteadas cinco direções; a densidade das partes vegetativas de árvores e arbustos de cada capão foi estimada pela média aritmética dos valores encontrados nos cinco transectos; (3) cobertura do dossel, obtida com um densiômetro, sendo sorteados cinco pontos no interior dos capões e, como a estimativa da cobertura da vegetação, foi utilizada a média aritmética dos valores encontrados nestes pontos; (4) altura máxima dos capões, obtida com um inclinômetro direcionado ao dossel de cada capão de mata, a uma distância de $120 \mathrm{~m}$ da borda.

Como não é possível testar todas as relações possíveis entre as variáveis independentes e as espécies de carabídeos - porque o número de testes excederia o número total de graus de liberdade, que é limitado pelo número de capões -, foram usadas técnicas de ordenação para reduzir a dimensionalidade com vetores associados às variáveis. Para obter os vetores associados às quatro variáveis que compõem a complexidade da vegetação, foi utilizada uma Análise de Componentes Principais (PCA). Os valores das variáveis da vegetação foram relativizados por divisão pela raiz quadrada da soma dos quadrados para eliminar o efeito das diferentes escalas.

A estrutura da comunidade de carabídeos foi representada por dois parâmetros: riqueza e composição de espécies. Como o número de besouros capturados em 
cada capão não pode ser controlado e a probabilidade de registrar novas espécies aumenta com este número, optou-se por estimar a riqueza de espécies em cada capão minimizando o efeito da abundância de besouros. Para tanto, a riqueza de espécies foi estimada por rarefação, através do programa EcoSim $7^{\mathrm{TM}}$ (GOTTELI \& ENTSMINGER, 2001), com 1.000 iterações aleatorizadas como amostras independentes.

A composição de espécies foi representada pelos vetores obtidos da ordenação dos capões pela abundância relativa de carabídeos. Escalonamento Multidimensional Híbrido (HMDS; FAITH et al., 1987) foi o método de ordenação adotado. O Escalonamento Multidimensional (MDS) não assume relações lineares entre variáveis, não depende de um índice de associação específico e freqüentemente resume mais informação em poucos vetores do que outros métodos de ordenação (MANLY, 2004). MDS resume as relações entre objetos através de uma matriz de distâncias baseada nas características dos objetos (e.g., os objetos são os capões e suas características, as abundâncias de cada espécie). Essa ordenação constrói uma configuração de pontos, que representam os objetos (capões neste estudo), em um número pré-determinado de dimensões (vetores), tanto que as diferenças entre objetos refletem as diferenças entre objetos na matriz de distâncias (MINCHIN, 1987). O índice de stress mede o quanto as distâncias medidas na matriz de associação diferem das diferenças entre objetos obtidas pela ordenação (MANLY, 2004). Entretanto, o índice de stress é indiretamente relacionado aos valores que medem os ajustes em outras técnicas de ordenação, então foi utilizado também o valor $\mathrm{r}^{2}$ de uma regressão linear entre os valores das distâncias de origem da matriz de associação e dos valores derivados da ordenação para uma, duas e três dimensões. O índice de dissimilaridade de Bray-Curtis (BRAY \& CuRTIS, 1957) foi utilizado para calcular as matrizes de distâncias entre capões baseadas nas abundâncias relativas de cada espécie.

Duas questões distintas e complementares foram abordadas: (1) se a riqueza de espécies está relacionada à inundação, ao tamanho e à complexidade da vegetação e (2) se a composição de espécies está relacionada a estas mesmas variáveis independentes. A inundação é uma variável categórica com dois níveis (capões inundáveis e capões não-inundáveis), o tamanho é uma variável contínua (perímetro em metros) e a complexidade da vegetação é representada pela interação entre os vetores da PCA. Na primeira questão, a riqueza de espécies é a única variável dependente e, para verificar a significância da relação com as variáveis independentes, foi usada análise de covariância. Na segunda questão, a composição de espécies é representada por até três vetores do HMDS (variáveis dependentes) e, para verificar a significância da relação com as variáveis independentes, foi aplicado o modelo de análise de covariância multivariada (LEGENDRE \& LEGENDRE, 1998).

\section{RESULTADOS}

Foram capturados 2.071 carabídeos de 64 espécies e 11 tribos (Tab. I). As tribos mais representativas em riqueza de espécies e em abundância foram Lebiini (975 besouros de 17 espécies) e Scaritini (481 de 15 espécies). Negrea scutellaris (Dejean, 1831) e Lebiini sp. 2 foram as espécies mais amostradas, com 472 e 464 indivíduos, respectivamente. Pheropsophus aequinoctialis (Linnaeus, 1763) (Brachinini) teve 95\% dos seus indivíduos coletados em abril e maio de 1999 e em apenas um dos capões de mata amostrados (Tab. I); nestes dois meses, $72 \%$ dos indivíduos desta espécie foram capturados em apenas cinco armadilhas, evidenciando sua distribuição agregada tanto no tempo, quanto no espaço. Em contraste, nos seis capões, 22 espécies foram representadas apenas por um indivíduo durante o período estudado, totalizando $34 \%$ da riqueza observada na comunidade.

A complexidade da vegetação foi representada pelos dois primeiros eixos da PCA pelas quatro variáveis ambientais - densidade de árvores e arbustos, cobertura e altura do dossel - que juntos capturaram 88,97 \% da variância nos dados (eigenvalue 3,677 para o eixo 1 e 1,667 para o eixo 2). O gradiente de complexidade da vegetação, utilizado como uma das variáveis para a análise dos dados, foi a interação entre estes dois eixos da PCA (Tab. II). O perímetro variou entre 425 e $535 \mathrm{~m}$ em capões inundáveis e entre 499 e 810 m em capões não-inundáveis.

A riqueza de espécies estimada por rarefação não diferiu entre os grupos de capões inundáveis e nãoinundáveis (Fig. 1). A análise de covariância indicou que a inundação e o tamanho dos capões não explicam a variação na estimativa de riqueza de espécies (Tab. III). Já a complexidade da vegetação foi um fator positivamente relacionado à riqueza de espécies. Mesmo com um pequeno número de amostras, a probabilidade desta relação não ser dada ao acaso foi bastante alta, com um valor marginalmente significativo ( $\mathrm{p}=0,067$; Tab. III).

A composição de espécies de carabídeos foi representada pelos dois vetores produzidos pela ordenação (HMDS) em duas dimensões dos capões de mata (Fig. 2). Esta ordenação registrou um índice de stress" de 0,156 e $\mathrm{r}^{2}$ de 0,859 , indicando que duas dimensões foram suficientes para capturar mais de $85 \%$ da variação na matriz de distâncias Bray-Curtis baseada na abundância relativa das espécies de besouros. A análise de covariância multivariada não detectou nenhuma relação significativa entre estes vetores da composição de espécies de carabídeos e as variáveis ambientais estudadas (Tab. IV).

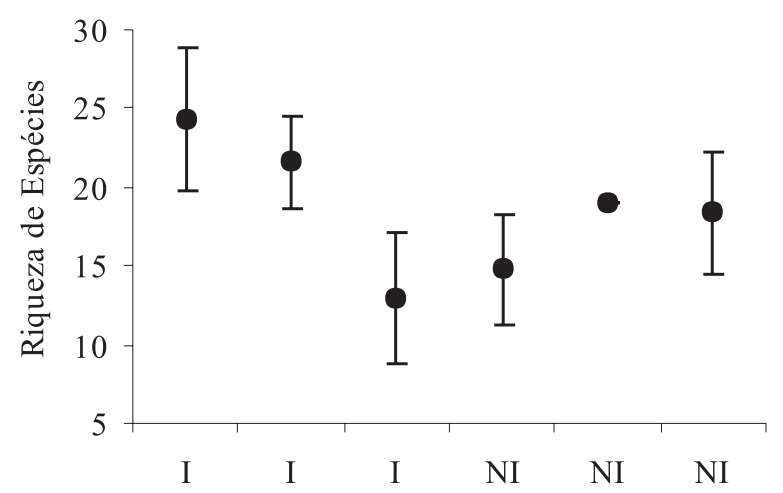

Fig. 1. Variação em riqueza de espécies de Carabidae (Coleoptera) em seis capões de mata das sub-regiões Miranda e Abobral no Pantanal sul-mato-grossense. Estimativas foram obtidas por rarefação para 133 indivíduos (menor número de indivíduos coletados que corresponde a um capão não-inundável). (I, capões inundáveis; NI, capões não-inundáveis. Barras indicam o intervalo de confiança de $95 \%$ das estimativas). 
Tabela I. Espécies de Carabidae (Coleoptera) registradas em capões de mata inundáveis (I) e não inundáveis (NI) das sub-regiões Miranda e Abobral no Pantanal sul-matogrossense. São dadas as abundâncias das espécies para cada capão e a frequiência (Freq) de cada espécie nos seis capões amostrados. Também são apresentados o número de espécies observado e o número de espécies esperado através do método de rarefação para 133 indivíduos.

\begin{tabular}{|c|c|c|c|c|c|c|c|c|}
\hline & \multicolumn{7}{|c|}{ Abundância por capão } & \multirow[b]{2}{*}{ Freq } \\
\hline & $\mathrm{I}$ & $\mathrm{I}$ & $\mathrm{I}$ & $\mathrm{NI}$ & NI & $\mathrm{NI}$ & Total & \\
\hline \multicolumn{9}{|l|}{ Bembidiini } \\
\hline Bembidiini sp.1 & 1 & 0 & 1 & 0 & 2 & 0 & 4 & 3 \\
\hline Bembidiini sp.2 & 0 & 1 & 0 & 1 & 0 & 0 & 2 & 2 \\
\hline Bembidiini sp. 3 & 0 & 1 & 1 & 1 & 0 & 1 & 4 & 4 \\
\hline Bembidiini sp.4 & 1 & 0 & 1 & 1 & 0 & 2 & 5 & 4 \\
\hline Bembidiini sp.5 & 0 & 0 & 1 & 0 & 0 & 0 & 1 & 1 \\
\hline Bembidiini sp.6 & 0 & 0 & 0 & 0 & 0 & 1 & 1 & 1 \\
\hline \multicolumn{9}{|l|}{ Brachinini } \\
\hline Pheropsophus aequinoctialis (Linnaeus, 1763) & 4 & 2 & 6 & 277 & 0 & 2 & 291 & 5 \\
\hline Brachinus sp. & 1 & 0 & 0 & 0 & 0 & 0 & 1 & 1 \\
\hline \multicolumn{9}{|l|}{ Cicindelini } \\
\hline Odontocheila brasiliensis (Dejean, 1825) & 3 & 8 & 4 & 2 & 4 & 0 & 21 & 5 \\
\hline \multicolumn{9}{|l|}{ Harpalini } \\
\hline Acupalpus sp. & 1 & 0 & 0 & 0 & 0 & 0 & 1 & 1 \\
\hline Harpalini sp.1 & 0 & 0 & 1 & 0 & 1 & 0 & 2 & 2 \\
\hline Harpalini sp. 2 & 1 & 0 & 2 & 6 & 1 & 2 & 12 & 5 \\
\hline Notiobia sp. & 2 & 0 & 0 & 0 & 0 & 2 & 4 & 2 \\
\hline Selenophorus sp. & 0 & 0 & 0 & 0 & 2 & 3 & 5 & 2 \\
\hline Stenolophus sp.1 & 0 & 0 & 1 & 1 & 0 & 1 & 3 & 3 \\
\hline Stenolophus sp.2 & 1 & 0 & 0 & 0 & 0 & 0 & 1 & 1 \\
\hline \multicolumn{9}{|l|}{ Lachnophorini } \\
\hline Lachnophorus sp. & 0 & 0 & 0 & 0 & 0 & 1 & 1 & 1 \\
\hline \multicolumn{9}{|l|}{ Lebiini } \\
\hline Calleida sp. & 0 & 0 & 1 & 0 & 0 & 1 & 2 & 2 \\
\hline Lebia sp. & 0 & 2 & 0 & 0 & 0 & 0 & 2 & 1 \\
\hline Lebiini sp.1 & 0 & 0 & 1 & 0 & 0 & 0 & 1 & 1 \\
\hline Lebiini sp.2 & 33 & 52 & 321 & 8 & 35 & 15 & 464 & 6 \\
\hline Lebiini sp.3 & 0 & 0 & 0 & 1 & 0 & 0 & 1 & 1 \\
\hline Lebiini sp.4 & 1 & 1 & 0 & 0 & 0 & 0 & 2 & 2 \\
\hline Lebiini sp.5 & 1 & 0 & 0 & 0 & 0 & 0 & 1 & 1 \\
\hline Lebiini sp.6 & 2 & 0 & 1 & 1 & 0 & 0 & 4 & 3 \\
\hline Lebiini sp.7 & 0 & 0 & 1 & 0 & 0 & 0 & 1 & 1 \\
\hline Lebiini sp. 8 & 0 & 1 & 0 & 4 & 4 & 3 & 12 & 4 \\
\hline Lebiini sp.9 & 0 & 0 & 0 & 1 & 0 & 0 & 1 & 1 \\
\hline Lebiini sp.10 & 0 & 2 & 1 & 0 & 0 & 0 & 3 & 2 \\
\hline Lebiini sp.11 & 1 & 0 & 0 & 0 & 0 & 0 & 1 & 1 \\
\hline Lebiini sp.12 & 0 & 0 & 0 & 0 & 0 & 1 & 1 & 1 \\
\hline Lebiini sp.13 & 0 & 1 & 0 & 0 & 1 & 0 & 2 & 2 \\
\hline Lebiini sp.14 & 1 & 0 & 0 & 0 & 1 & 1 & 3 & 3 \\
\hline Lebiini sp.15 & 0 & 1 & 1 & 0 & 0 & 0 & 2 & 2 \\
\hline Negrea scutellaris (Dejean, 1831) & 22 & 52 & 156 & 23 & 29 & 190 & 472 & 6 \\
\hline \multicolumn{9}{|l|}{ Masoreini } \\
\hline Macracanthus (Macracanthus) sp.1 & 40 & 5 & 6 & 4 & 10 & 1 & 66 & 6 \\
\hline Macracanthus (Macracanthus) sp. 2 & 9 & 1 & 0 & 1 & 0 & 0 & 11 & 3 \\
\hline \multicolumn{9}{|l|}{ Megacephalini } \\
\hline Megacephala brasiliensis (Kirby, 1818) & 0 & 0 & 1 & 0 & 0 & 0 & 1 & 1 \\
\hline \multicolumn{9}{|l|}{ Pterostichini } \\
\hline Loxandrus sp.1 & 3 & 0 & 0 & 0 & 0 & 0 & 3 & 1 \\
\hline Loxandrus sp.2 & 1 & 3 & 1 & 5 & 0 & 0 & 10 & 4 \\
\hline Pterostichina sp.1 & 6 & 0 & 0 & 0 & 1 & 0 & 7 & 2 \\
\hline Pterostichina sp.2 & 2 & 1 & 2 & 3 & 0 & 15 & 23 & 5 \\
\hline Pterostichina sp.3 & 30 & 1 & 3 & 2 & 5 & 13 & 54 & 6 \\
\hline Pterostichini sp.1 & 1 & 0 & 0 & 0 & 0 & 0 & 1 & 1 \\
\hline Pterostichini sp.2 & 1 & 0 & 0 & 0 & 0 & 0 & 1 & 1 \\
\hline Pterostichini sp.3 & 4 & 1 & 1 & 0 & 0 & 0 & 6 & 3 \\
\hline Tichonilla sp. & 57 & 6 & 2 & 0 & 0 & 3 & 68 & 4 \\
\hline Scaritini & & & & & & & & \\
\hline Aspidoglossa sp.1 & 23 & 12 & 28 & 16 & 19 & 19 & 117 & 6 \\
\hline Aspidoglossa sp.2 & 22 & 2 & 0 & 0 & 0 & 2 & 26 & 3 \\
\hline Aspidoglossa sp. 3 & 0 & 0 & 0 & 0 & 0 & 1 & 1 & 1 \\
\hline Camptodontus sp. & 3 & 0 & 1 & 0 & 2 & 0 & 6 & 3 \\
\hline Clivina sp.1 & 50 & 10 & 98 & 6 & 9 & 9 & 182 & 6 \\
\hline Clivina sp.2 & 1 & 0 & 1 & 0 & 0 & 0 & 2 & 2 \\
\hline Clivina sp.3 & 0 & 2 & 0 & 0 & 0 & 0 & 2 & 1 \\
\hline Clivina sp.4 & 12 & 0 & 6 & 8 & 3 & 0 & 29 & 4 \\
\hline Mesus pseudogigas (Vieira \& Bello, 2004) & 0 & 0 & 0 & 0 & 0 & 1 & 1 & 1 \\
\hline
\end{tabular}


Tabela I (cont.)

\begin{tabular}{lccccccc}
\hline & \multicolumn{1}{c}{ Abundância por capão } \\
\cline { 2 - 6 } & I & I & I & NI & NI & NI & Total \\
Freq
\end{tabular}

Tabela II. Médias dos valores da cobertura do dossel, número de árvores e arbustos, perímetro e altura máxima dos capões inundáveis (I) e não inundáveis (NI) amostrados. São dados os valores dos eixos 1 e 2 da análise de componentes principais (PCA), que representam a variação da complexidade vegetacional dos capões neste estudo. Para mais detalhes, vide Material e Métodos.

\begin{tabular}{lccccccc}
\hline Capão & $\begin{array}{c}\text { Média da } \\
\text { cobertura }(\%)\end{array}$ & $\begin{array}{c}\text { Média do } \\
\mathrm{n}^{\circ} \text { arbustos }\end{array}$ & $\begin{array}{c}\text { Média do } \\
\mathrm{n}^{\circ} \text { árvores }\end{array}$ & $\begin{array}{c}\text { Altura } \\
(\mathrm{m})\end{array}$ & \multicolumn{2}{c}{ Complexidade da vegetação } & \multicolumn{2}{c}{$\begin{array}{c}\text { Perímetro } \\
\text { PCA } 1\end{array}$} & $\begin{array}{c}\text { PCA2 } \\
(\mathrm{m})\end{array}$ \\
\hline $\mathrm{I}$ & 89,500 & 20,833 & 2,333 & 12 & $-0,748$ & 0,659 & 535 \\
I & 83,833 & 21,333 & 3,667 & 14 & 0,992 & 0,075 & 425 \\
I & 85,000 & 16,333 & 4,000 & 15 & $-0,621$ & $-0,554$ & 535 \\
NI & 86,000 & 11,833 & 3,333 & 8 & 0,152 & $-0,944$ & 499 \\
NI & 87,667 & 22,000 & 3,500 & 10 & $-0,863$ & 0,063 & 705 \\
NI & 59,833 & 12,333 & 1,667 & 12 & 0,990 & 0,136 & 810 \\
\hline
\end{tabular}

Tabela III. Análise de covariância entre a riqueza de espécies estimada pelo método de rarefação para 133 indivíduos e as fontes de variação estudadas $\left(n=6 ; r^{2}=0,886\right)$.

\begin{tabular}{lcc}
\hline Fonte & $\mathrm{F}$ & $\mathrm{p}$ \\
\hline Tamanho & 0,353 & 0,613 \\
Inundação & 0,246 & 0,669 \\
Complexidade vegetacional & 13,395 & 0,067 \\
\hline
\end{tabular}

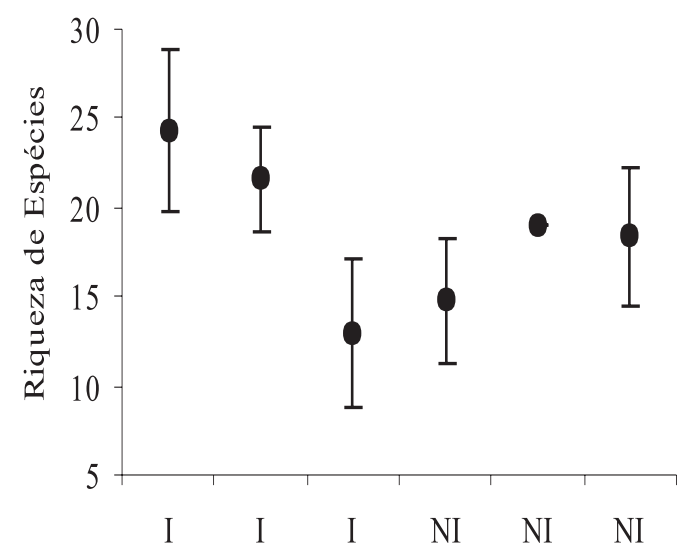

Fig. 2. Ordenação por HMDS em duas dimensões (stress=0,156 e $\left.\mathrm{r}^{2}=0,859\right)$ em seis capões de mata nas sub-regiões Miranda e Abobral, Pantanal sul-mato-grossense (I, capões inundáveis; NI, capões não inundáveis).

\section{DISCUSSÃO}

Os capões de mata no Pantanal sul-mato-grossense são compostos por um elevado número de espécies raras e poucas espécies dominantes de carabídeos. A ecologia e a taxonomia destes besouros em florestas temperadas
Tabela IV. Análise de covariância multivariada (MANCOVA) entre a composição de Carabidae, representada pela ordenação dos seis capões amostrados e as variáveis ambientais amostradas.

\begin{tabular}{lccc}
\hline Fonte & Pillai Trace & $\mathrm{F}$ & $\mathrm{P}$ \\
\hline Tamanho & 0,616 & 0,801 & 0,620 \\
Inundação & 0,657 & 0,956 & 0,586 \\
Complexidade vegetacional & 0,651 & 0,932 & 0,591 \\
\hline
\end{tabular}

é bem conhecida (Molnár et al., 2001; PAARMAnn et al., 2001; LÖVEI \& SUDERLAND, 1996), sendo as armadilhas de queda ideais para sua captura (Lindroth, 1974 apud PAARMANn et al., 2001). Em contraste, na região tropical, carabídeos coletados por estas armadilhas registram elevada riqueza, porém são pouco abundantes (HAMMOND, 1990), isto porque apresentam distribuição gregária em resposta ao padrão de disponibilidade de recursos (PAARMANN et al., 2001).

Pheropsophus aequinoctialis exibiu uma distribuição gregária evidente, fato já observado por REICHARDT (1971), que afirma que esta espécie ocorre habitualmente em grandes agregações que permanecem durante o dia sob pedras e troncos caídos. $\mathrm{O}$ registro desta espécie foi expressivo nos meses de março e abril de 1999, com 95\% dos indivíduos capturados em apenas um capão. Pheropsophus rivieri Demay, 1838, não registrada neste estudo, apresenta um ciclo de vida adaptado ao regime de inundação das planícies da Amazônia Central e é encontrada em agregações nos três primeiros meses da vazante, exibindo um padrão de atividade sazonal (ZeRM \& AdIS, 2004). Espécies de carabídeos em bordas de mata e campos também exibem um padrão de agregação, que é persistente e localizada nos campos durante uma estação; cada espécie apresenta um comportamento gregário diferente e estabilidade dos grupos distinta (THомas et al., 2001). 
Além da distribuição agregada descrita para os trópicos, a captura de espécies raras de carabídeos é afetada por dois fatores (BUTTERFIELD \& Coulson, 1983): 1) a captura depende do acaso e 2) a alta atividade de deslocamento de muitas espécies resulta em inúmeras capturas de espécies vágeis e ocasionais em locais que não são seu hábitat permanente. Como exemplo, as espécies de Bembidiini - que vivem em troncos e sob cascas de árvores (REICHARDT, 1977; ERWIN \& SimS, 1984) -, foram registradas eventualmente, assim como Mesus pseudogigas Vieira \& Bello, 2004, cujos possíveis hábitats seriam as matas ciliares e não os capões (VIEIRA \& BELLO, 2004). Portanto, os capões de mata são ambientes transitórios para muitas espécies.

Outro exemplo de captura ocasional é o de representantes da tribo Harpalini (e.g. Notiobia sp. e Selenophorus sp.), que são predadores de sementes e frutos e vivem no folhiço sob gramíneas (MARINONI et al., 2001). Nas florestas de terra firme da Amazônia Central, as larvas de Notiobia se desenvolvem em sementes de Ficus e Cecropia (ARndt, 1998), gêneros de plantas freqüentes nos capões de mata (Ротт \& Ротт, 1994). Para evitar a competição após a queda dos frutos, os adultos deslocam-se para outros ambientes (PAARMAN et al., 2001) e portanto, a ocorrência deste gênero nas armadilhas está relacionada com sua busca por recursos.

Neste estudo, a complexidade vegetacional dos capões de mata foi representada pela ordenação (PCA) de quatro variáveis (densidade de árvores e arbustos, altura do capão e cobertura do dossel) relacionadas com a estrutura física do ambiente em questão, pois a composição de espécies de plantas por si só não está diretamente relacionada com as mudanças espaciais na composição de espécies animais (e.g. MACARThUR \& MacArthur, 1961; Quinn et al., 1991; Tews, 2004). A estrutura da vegetação e suas modificações derivadas em microclima é um dos fatores mais importantes na distribuição dos carabídeos (Molnár et al., 2001). Vários estudos confirmam que a heterogeneidade vegetacional está positivamente relacionada à diversidade destas espécies (BEDFORD \& UsCheR, 1994; NimIELÄ, 1997) e, em ambientes homogêneos como as florestas de coníferas, a riqueza de espécies é naturalmente menor (FAHY \& Gormally, 1998).

Apesar de diversos estudos comprovarem a relação entre a complexidade da vegetação e a composição de espécies de Carabidae em agroecossistemas (QuinN et al., 1991; Lys et al., 1994; VARCHOLA \& DunN, 1999, 2001; FRENCH \& ELLIOTT, 1999) e em ambientes naturais (NIMIELÄ, 1990b; Crist \& Ahern, 1999; INGS \& HartLey, 1999; Molnár et al., 2001), a comunidade de carabídeos nos capões não está estruturada pela complexidade vegetacional. A variação na complexidade vegetacional nos capões não foi tão expressiva quanto em diferentes sistemas de cultivo e em outras formações florestais, o que pode mascarar este efeito.

Uma das predições da teoria do equilíbrio da biogeografia de ilhas é que em ilhas maiores há mais espécies do que em ilhas menores (MacArthur \& Wilson, 1967). Nos capões de mata estudados, que correspondem a ilhas de vegetação para espécies intolerantes à inundação e às condições inerentes à matriz campestre que os permeia, tal previsão não foi confirmada. Este é um resultado inesperado, uma vez que o tamanho dos capões limita a ocorrência das espécies de carabídeos, uma vez que as áreas individuais de atividade estão relacionadas com o tamanho corporal destes besouros (DEN BoER, 1989). Portanto, sugere-se que os capões não representam ilhas para os carabídeos e a matriz campestre não representa barreira à sua dispersão.

As necessidades ambientais requeridas pelos carabídeos são bem conhecidas, mas as relações entre as variáveis ambientais e os padrões observados na abundância e distribuição das espécies não (NIMIELÄ, 1996). A ocorrência das espécies é parcialmente relacionada a diversas variáveis ambientais que operam em diferentes escalas espaciais, contudo, os fatores que operam em uma escala tem pouco ou nenhum efeito em outra (NIMIELÄ, 1996).

Agradecimentos. À CAPES, pela concessão da bolsa de mestrado à L. Vieira. Ao CNPq, pelo apoio financeiro ao projeto integrado de pesquisa (processo 521746/97-3) e pela bolsa de desenvolvimento científico e regional de J. Raizer (processo 351235/97-3). Ao Sr. Ayr de M. Bello pelo auxílio na identificação dos carabídeos. Aos doutores Helena C. de Morais, Priscila P. Lopes, Júlio N. C. Louzada, Carlos F. Sperber, Marcel O. Tanaka e Fernando Paiva, pelas sugestões e críticas ao trabalho. À Elizabeth Nichols pelas sugestões ao abstract.

\section{REFERÊNCIAS BIBLIOGRÁFICAS}

Arndt, E. 1998. The species of Notiobia Perty (Coleoptera: Carabidae: Harpalini) from Brazil. Acta Amazonica 28(3):285-299.

Bedford, S. E. \& Uscher, M. B. 1994. Distribution of arthropod species across the margins of farm woodlands. Agriculture, Ecosystems and Environment 48:295-305.

Воотн, R. G.; Cox, M. L. \& Magde, R. B. 1990. IIE Guide to insects of importance to man: 3. Coleoptera. Wallingford, CAB International. 384p.

Bray, J. R. \& Curtis, J. T. 1957. An ordination of the upland forest communities of southern Wisconsin. Ecological Monographs 27:325-349.

Butterfield, J. \& Coulson, J. C. 1983. The carabid communities on peat and upland grassland in northern England (U. K.). Holartic Ecology 6(2):163-174.

Crist, T. O. \& AHERn, R. G. 1999. Effects of habitat patch size and temperature on the distribution and abundance of ground beetles (Coleoptera: Carabidae) in an old field. Environmental Entomology 28(4):680-689.

Davidowitz, G. \& Rosenzweig, M. L. 1998. The latitudinal gradient of species diversity among North American grasshoppers within a single habitat: a test of the spatial heterogeneity hypothesis. Journal of Biogeography 25:553-560.

Den Boer, P. J. 1989. Comment on the article "On testing temporal niche differentiation in carabid beetles" by M. Loreau. Oecologia 81:97-98.

Diefenbach, L. M. G. \& Becker, M. 1992. Carabid taxocenes of an urban park in subtropical Brazil: I. Specific composition, seasonality and constancy (Insecta: Coleoptera: Carabidae). Studies on Neotropical Fauna and Environment 27(4):169-187.

ERwin, T. \& Sims, L. L. 1984. Carabid beetles of the West Indies (Insects: Coleoptera): A synopsis of the genera and checklists of tribes of Caraboidea, and of the West Indian species. Quaestiones Entomologicae 20:351-466.

Eyre, M. D.; Luff, M. L.; Stanley, J. R. \& Telfer, M. G. 2003. The relationship between British ground beetles (Coleoptera, Carabidae) and land cover. Journal of Biogeography 30:719-730 
FAHRIG, L. 2003. Effects of habitat fragmentation on biodiversity. Annual Review of Ecology, Evolution and Systematics 34:487-515.

Fahy, O. \& GoRmally, M. 1998. A comparison of plant and carabid beetle communities in an Irish oak woodland with a nearby conifer plantation and clear felled site. Forest Ecology and Management 110:263-273.

Faith, D. P.; Minchin, P. R. \& Belbin, L. 1987. Compositional dissimilarity as a robust measure of ecological distance. Vegetatio 69:57-68.

French, W. B. \& Elliott, N. C. 1999. Spatial and temporal distribution of ground beetle (Coleoptera: Carabidae) assemblages in riparian strips and adjacent wheat fields. Environmental Entomology 28(4):597-607.

Gotelli, N. J. \& Entsminger, G. L. 2001. EcoSim: Null models software for ecology. Version 7. Acquired Intelligence Inc. \& Kesey-Bear. Burlington, VT05465. Disponível em: <http:// homepages.together.net/ gentsmin/ecosim.htm>. Acesso em: 15.08.2003

Hammond, P. 1990. Concluding remarks: Carabids in context. In: Stork, N. E. ed. The role of ground beetles in ecological and environmental studies. Andover, Intercept Limited. p.403-409.

IngS, T. C. \& HartLEy, S. E. 1999. The effect of habitat structure on carabid communities during the regeneration of a native Scottish forest. Forest, Ecology and Management 119:123-136.

JunK, W. J. 1993. Wetlands of Tropical South America. In: Whigham, D. J.; Dykyjorá, D. \& Hejny, S. eds. Wetlands of the World: inventory, ecology and management. USA, Library of Congress. v.1, p.679-739.

Lawton, J. H. 1983. Plant architecture and the diversity of phytophagous insects. Annual Review of Entomology 28:23-39.

Legendre, P. \& Legendre, L. 1998. Numerical ecology. 2 ed. Amsterdam, Elsevier Scientific Publishing Company. 870p.

Lövei, G. L. \& SunderLand, K. D. 1996. Ecology and behavior of ground beetles (Coleoptera: Carabidae). Annual Review of Entomology 41:231-256.

Lys, J.-A.; Zimmermann, M. \& Nentwig, W. 1994. Increase in activity density and species number of carabid beetles in cereals as a result of strip-management. Entomologia Experimentalis et Applicata 73:1-9.

MacArthur, R. H. \& MacArthur, J. W. 1961. On bird species diversity. Ecology 42:594-598.

MacArthuR, R. H. \& Wilson, E. O. 1967. The theory of island biogeography. Princeton, NJ, Princeton University Press. 203 p.

MANLY, B. J. 2004. Multivariate statistical methods: a primer. 3 ed. New York, Chapman and Hall. 214p.

Marinoni, R. C.; Ganho, N. G.; Monné, M. L. \& Mermudes, J. R. M. 2001. Hábitos alimentares em Coleoptera (Insecta): organização, compilação, organização de dados e novas informações sobre alimentação nas famílias de coleópteros. Ribeirão Preto, Holos. 64p.

McCoy, E. D. \& BeLl, S. S. 1991. Habitat structure: the evolution and diversification of a complex topic. In: BeLL, S. S.; McCoy, E. D. \& Mushinsky, H. R. eds. Habitat structure: the physical arrangement of objects in space. London, Chapman and Hall. p.3-27.

Minchin, P. R. 1987. An evaluation of the relative robustness of techniques for ecological ordination. Vegetatio 69:89-107.

Molnár, T.; Magura, T.; Tóthmérész, B. \& Elek, Z. 2001. Ground beetles (Carabidae) and edge effect in oak-hornbeam forest and grassland transects. European Journal of Soil Biology 37:297-300.

NimielÄ, J. 1990a. Habitat distribution of carabid beetles in Tierra del Fuego, South America. Entomologica Fennica 1:3-16. 1990b. Spatial distribution of carabid beetles in the southern Finnish taiga: a question of scale. In: STORK, N. E. ed. The role of ground beetles in ecological and environmental studies. Andover, Intercept Limited. p.143-155.

1996. From systematics to conservation - carabidologists do it all. Annales Zoologici Fennici 33:1-4.

1997. Invertebrates and boreal forest management. Conservation Biology 11:601-610.
Noonan, G. R. 1985. Classification and names of the Selenophorini group (Coleoptera: Carabidae: Harpalini) and of nine genera and subgenera placed in incertae sedis within Harpalina. Contributions in Biology and Geology 64:1-92.

Oliveira-FilHo, A. T. 1992. Floodplain 'murundus' of Central Brazil: evidence for the termite-origin hypothesis. Journal of Tropical Ecology 8:1-19.

Paarmann, W.; Adis, J.; Stork, N.; Gutzmann, B.; Stumpe, P.; Staritz, B.; Bolte, H.; Küppers, S.; Holzkamp, K.; Niers, C. \& Fonseca, C. R. V DA. 2001. The structure of ground beetle assemblages (Coleoptera: Carabidae) at fig fruit falls (Moraceae) in terra firme rain forest near Manaus (Brazil). Journal of Tropical Ecology 17:549-561.

Pavuk, D. M.; Purrington, F. F.; Willians, C. E. \& Stinner, B. R 1997. Ground beetle (Coleoptera: Carabidae) activity density and community composition in vegetationally diverse corn agrossystems. The American Midland Naturalist 138: $14-28$

Ponce, V. M. \& Cunha, C. N. 1993. Vegetated earthmounds in tropical savannas of Central Brazil: a synthesis. Journal of Biogeography 20:219-225.

Ротт, A. \& Ротт, V. J. 1994. Plantas do Pantanal. Brasília, Embrapa. 320p.

Prance, G. T. \& Schaller, G. B. 1982. Preliminary study of some vegetation types of the Pantanal, Mato Grosso, Brazil. Brittonia 34:228-251.

Quinn, M. A.; Kepner, R. L.; Walgenbach, D. D.; Nelson Foster, R. Bohls, R. A.; Pooler, P. D; Reuter, K. C. \& Swain, J. L. 1991. Effect of habitat characteristics and perturbation from insecticides on the community dynamics of ground beetles (Coleoptera: Carabidae) on mixed-grass Rangeland. Environmental Entomology 20(5):1285-1294.

REICHARDT, H. 1971. Notes of the bombarding behavior of three carabid beetles (Coleoptera, Carabidae). Revista Brasileira de Entomologia 15(5):31-34.

1974. Revision of Mesus Chevrolat, 1858, with the description of three new species (Coleoptera, Carabidae). Revista Brasileira de Entomologia 18:77-84.

1977. Synopsis of the genera of Neotropical Carabidae (Insecta: Coleoptera). Quaestiones Entomologicae 13:346-493

Roig-Juñent, S. \& Domínguez, M. C. 2001. Diversidad de la familia Carabidae (Coleoptera) en Chile. Revista Chilena de Historia Natural 74:549-571.

Silva, M. P.; Mauro, R.; Mourão, G. \& Coutinho, M. 2000. Distribuição e quantificação de classes de vegetação do Pantanal através de levantamento aéreo. Revista Brasileira de Botânica 23(2):143-152.

Straneo, S. L. 1979. Notes about classification of the south american Pterostichini with a key for determination of subtribes, genera and subgenera (Coleoptera: Carabidae). Quaestiones Entomologicae 15:345-356.

Sullivan, T. P. \& Sullivan, D. S. 2001. Influence of variable retention harvests on forest ecosystems. II. Diversity and population dynamics of small mammals. Journal of Applied Ecology 38:1234-1252.

Tews, J.; Brose, U.; Grimm, V.; Tielböerger, K.; Wichmann, M. C. Schwager, M. \& Jeltsch, F. 2004. Animal species diversity driven by habitat heterogeneity/diversity: the importance of keystone structures. Journal of Biogeography 31:79-92.

Thiele, H. U. 1977. Carabid beetles in their environments: a study on habitat selection by adaptation in physiology and behaviour. Berlin, Spring-Verlag. 369p.

Thomas, C. F. G.; Parkinson, L.; Griffiths, G. J. K.; Fernandez Garcia, A. \& Marshall, E. J. P. 2001. Aggregation and temporal stability of carabid beetle distributions in field and hedgerow habitats. Journal of Applied Ecology 38:100-116.

Varchola, J. M. \& DunN, J. P. 1999. Changes in ground beetle (Coleoptera: Carabidae) assemblages in farming systems bordered by complex or simple roadside vegetation. Agriculture, Ecosystems and Environment 73:41-49. 2001. Influence of hedgerow and grassy field borders on ground beetle (Coleoptera: Carabidae) activity in fields of corn. Agriculture, Ecosystems and Environment 83:153-163.

Vieira, L. M. \& Bello, A. M. 2004. Uma nova espécie do gênero Mesus Chevrolat do Mato Grosso do Sul, Brasil (Coleoptera, 
Carabidae, Scaritini). Revista Brasileira de Entomologia 48(2): 243-245

Zerm, M. \& Adis, J. 2004. Survival strategy of the bombadier beetle, Pheropsophus rivieri (Col.: Carabidae) in a Central Amazonian blackwater floodplain (Brazil). Amazoniana 17(3/4):503-508

Recebido em setembro de 2006. Aceito em dezembro de 2007. ISSN 0073-4721

Artigo disponível em: www.scielo.br/isz

Iheringia, Sér. Zool., Porto Alegre, 98(3):317-324, 30 de setembro de 2008 